\title{
The Optimum Addition of Points to Quadrature Formulae*
}

\author{
By T. N. L. Patterson
}

Abstract. Methods are developed for the addition of points in an optimum manner to the Gauss, Lobatto and general quadrature formulae. A new set of $n$-point formulae are derived of degree $(3 n-1) / 2$.

1. Introduction. In a recent book Kronrod [1] has shown how the $n$-point Gaussian quadrature formulae may be augmented by a set of $n+1$ abscissae to yield quadrature formulae of degree $3 n+1$ ( $n$ even) or $3 n+2$ ( $n$ odd). The importance of these formulae is that the accuracy of a numerical integration can be considerably improved without wasting the integrand evaluations at the Gaussian abscissae. Kronrod has given tables of these extended abscissae including their associated weights for all Gaussian formulae up to 40 points.

Kronrod noted that as $n$ is increased a large number of guarding digits have to be carried to preserve the accuracy of the results and implied that about sixtyfive decimal digits were carried to produce the tables correct to sixteen decimal places. It is, unfortunately, the large values of $n$ which are likely to be of the most interest.

In this paper it is shown how the additional abscissae may be derived in a numerically stable fashion by an expansion of the equation for the abscissae in terms of Legendre polynomials. A technique is also discussed to extend the $n$-point Lobatto quadrature formulae by the addition of $n-1$ abscissae to yield quadrature formulae of degree $3 n-3$ ( $n$ even) or $3 n-2$ ( $n$ odd). Finally a method is discussed for the optimum addition of abscissae to general quadrature formulae and a new set of $n$-point formulae is derived of degree $(3 n-1) / 2$.

2. The Extension of Quadrature Formulae. The basic reasoning behind the extension of quadrature formulae is as follows. Let an $n$-point formula be augmented by the addition of $p$ abscissae and let $G_{n+p}(x)$ be the polynomial whose roots are the $n+p$ abscissae of the new quadrature formula. A general polynomial of degree $n+2 p-1$ can be expressed as

$$
F_{n+2 p-1}(x)=Q_{n+p-1}(x)+G_{n+p}(x) \sum_{k=0}^{p-1} c_{k} x^{k}
$$

where $Q_{n+p-1}(x)$ is a general polynomial of degree $n+p-1$. This transformation of $F_{n+2 p-1}(x)$ is possible since the number of unknown coefficients on the left- and right-hand sides of (1) is equal. $Q_{n+p-1}(x)$ can always be exactly integrated by a $(n+p)$-point formula and if $G_{n+p}(x)$ is such that

Received December 1, 1967.

* This research was supported by the National Aeronautics and Space Administration under Grant NSG 269. 


$$
\int_{-1}^{1} G_{n+p}(x) x^{k} d x=0, \quad k=0,1, \cdots, p-1
$$

then all of (1) can be exactly integrated by an $(n+p)$-point quadrature formula. Thus it should, in principle, be possible to derive formulae having $n+p$ abscissae and of degree $n+2 p-1$.

2.1. The Extension of the Gauss Formulae. Kronrod [1] has considered the case $p=n+1$ for the $n$-point Gauss formula. This choice of $p$ yields the number of points required to subdivide the intervals spanned by the $n$ original Gauss points. The resulting quadrature formula should have degree $3 n+1$. Since the formulae are symmetrical in the range $[-1,1]$ odd functions are always integrated exactly with value zero. Hence the effective degree can be increased to $3 n+2$ when $n$ is odd. For this choice of $p$ the polynomial $K_{n+1}(x)$ whose $n+1$ roots are the additional abscissae must satisfy, corresponding to (2),

$$
\int_{-1}^{1} K_{n+1}(x) P_{n}(x) x^{k} d x=0 \text { for } k=0,1, \cdots, n
$$

where $P_{n}(x)$ is the Legendre polynomial. Kronrod determines $K_{n+1}(x)$ and hence its zeros by substituting its polynomial expansion into (3) and solving the resulting triangular system of equations to find the polynomial coefficients. It is at this point that the numerical difficulties arise. When $n$ is large, the polynomial coefficients of $K_{n+1}(x)$ differ greatly in magnitude, so the significant inaccuracies due to both rounding and cancellation errors can appear in their calculation and when they are used to evaluate $K_{n+1}(x)$.

These numerical difficulties can be circumvented by expanding $K_{n+1}(x)$ in terms of orthogonal polynomials, in particular the Legendre polynomials. Writing

$$
K_{n+1}(x)=\sum_{i=1}^{r} a_{i} P_{2 i-1-q}(x),
$$

where $[x]$ denotes the integer part of $x, q=n-2[n / 2]$ and $r=[(n+3) / 2]$, then (3) becomes

$$
\sum_{i=1}^{r} a_{i} \int_{-1}^{1} P_{2 i-1-q}(x) P_{n}(x) x^{k} d x=0 .
$$

Since the points should be added symmetrically (that is, if $x$ is an abscissa then so is $-x) K_{n+1}(x)$ must be an odd or an even function and can be expressed in the form (4). The notation insures that odd and even values of $n$ are correctly dealt with. Since $x^{k}$ can be expanded in terms of Legendre polynomials and vice versa, condition (5) can also be expressed with $x^{k}$ replaced by $P_{k}(x)$. In addition, since odd functions automatically satisfy (5) because of symmetry, then only odd values of $k$ in (5) need be considered (note that $P_{2 i-1-q}(x) P_{n}(x)$ is an odd function). Thus (5) finally becomes

$$
\sum_{i=1}^{r} a_{i} \int_{-1}^{1} P_{2 i-1-q}(x) P_{n}(x) P_{2 k-1}(x) d x=0, \quad k=1,2, \cdots, r-1 .
$$

Writing 


$$
S_{i, k}=\int_{-1}^{1} P_{2 i-1-q}(x) P_{n}(x) P_{2 k-1}(x) d x,
$$

then (6) can be expressed as

$$
\sum_{i=1}^{r} a_{i} S_{i, k}=0, \quad k=1,2, \cdots, r-1 .
$$

It can be shown [2] that

$$
S_{i, k}=0 \quad \text { if } i+k<r .
$$

Equation (8) then becomes

$$
\sum_{i=r-k}^{r} a_{i} S_{i, k}=0
$$

or expanding in full

$$
\begin{aligned}
a_{r-1} & =-a_{r} \frac{S_{r, 1}}{S_{r-1,1}}, \\
a_{r-2} & =-a_{r} \frac{S_{r, 2}}{S_{r-2,2}}-a_{r-1} \frac{S_{r-1,2}}{S_{r-2,2}}, \\
& \cdot \\
& \cdot \\
& \cdot \\
& \cdot \\
a_{1} & =-a_{r} \frac{S_{r, r-1}}{S_{1, r-1}}-a_{r-1} \frac{S_{r-1, r-1}}{S_{1, r-1}} \cdots-a_{2} \frac{S_{2, r-1}}{S_{1, r-1}},
\end{aligned}
$$

which can be solved recursively. The coefficient $a_{r}$ can be arbitrarily set equal to 1 without affecting the calculation of the roots of $K_{n+1}(x)$. It can be shown that

(12) $\frac{S_{i, k}}{S_{r-k, k}}$

Thus the quantities appearing in (11) can be recursively calculated using (12) with $i=r+1-k, \cdots, r$ in steps of one for each of $k=1,2, \cdots, r-1$. Fven for high values of $n$ the $a_{i}$ do not vary excessively in magnitude and in calculating the roots of $K_{n+1}(x)$ very few digits are lost through cancellation and round-off. For example, using sixteen-decimal-digit arithmetic at most two digits were lost for the case of $n=65$.

The expansion in terms of Legendre polynomials can easily be summed by a simple algorithm which is based on the recurrence properties of the polynomials. To evaluate $S=\sum_{j=0}^{n} a_{j} P_{j}(x)$, a series of coefficients $b_{j}$ is calculated from the recurrence relation

$$
b_{j}=\left\{(2 j+1) x b_{j+1}-(j+1) b_{j+2}-a_{j}\right\} / j
$$

for $j=n, n-1, \cdots, 1$ with $b_{n+1}=b_{n+2}=0$. Then $S=a_{0}+b_{2}-b_{1} x$. 
2.2. The Extension of Lobatto Formulae. The addition of $n-1$ points $(p=n-1)$ to the $n$-point Lobatto formula should allow the derivation of a quadrature formula of degree $3 n-3$ ( $n$ even) or $3 n-2(n$ odd $)$. This choice of $p$ gives sufficient points to subdivide the intervals spanned by the original $n$ abscissae.

Noting that the $n$ Lobatto abscissae are the roots of the polynomial $\left(x^{2}-1\right) P_{n-1}^{\prime}(x)$ the polynomial $W_{n-1}(x)$ whose roots are the required additional points must satisfy, corresponding to (2),

$$
\int_{-1}^{1} W_{n-1}(x)\left(x^{2}-1\right) P_{n-1}^{\prime}(x) x^{k} d x=0, \quad k=0,1, \cdots, n-2 .
$$

Again, as discussed earlier, $x^{k}$ may be replaced by $P_{k}(x)$ and taking account of symmetry and the recurrence relations between the Legendre polynomials and their derivatives (14) may be reduced to,

$$
\begin{aligned}
\sum_{i=1}^{r-1} g_{i} \int_{-1}^{1} P_{2 i-1-q}(x)\left\{P_{n}(x)-P_{n-2}(x)\right\} P_{2 k-1}(x) d x & =0, \\
k & =1,2, \cdots, r-2,
\end{aligned}
$$

where

$$
W_{n-1}(x)=\sum_{i=1}^{r-1} g_{i} P_{2 i-1-q}(x),
$$

the expansion again being in terms of Legendre polynomials. As before $q=$ $n-2[n / 2]$ and $r=[(n+3) / 2]$. Defining

$$
\begin{aligned}
& S_{i, k}=\int_{-1}^{1} P_{n}(x) P_{2 i-1-q}(x) P_{2 k-1}(x) d x, \\
& D_{i, k}=\int_{-1}^{1} P_{n-2}(x) P_{2 i-1-q}(x) P_{2 k-1}(x) d x,
\end{aligned}
$$

and

$$
U_{i, k}=S_{i, k}-D_{i, k},
$$

then Eq. (15) reduces to

$$
\sum_{i=1}^{r-1} g_{i} U_{i, k}=0, \quad k=1,2, \cdots, r-2 .
$$

It can be shown that

$$
U_{i, k}=0 \text { if } i+k<r-1 .
$$

'Thus (20) becomes

$$
\sum_{i=r-1-k}^{r-1} g_{i} U_{i, k}=0, \quad k=1,2, \cdots, r-2 .
$$

or 


$$
\begin{aligned}
& g_{r-2}=-g_{r-1} \frac{U_{r-1,1}}{U_{r-2,1}}, \\
& g_{r-3}=-g_{r-1} \frac{U_{r-1,2}}{U_{r-3,2}}-g_{r-2} \frac{U_{r-2,2}}{U_{r-3,2}},
\end{aligned}
$$

$$
g_{1}=-g_{r-1} \frac{U_{r-1, r-2}}{U_{1, r-2}}-\cdots-g_{2} \frac{U_{2, r-2}}{U_{1, r-2}}
$$

Writing

$$
\frac{U_{i, k}}{U_{i-1, k}}=\frac{D_{i, k}}{D_{i-1, k}} \frac{\left\{S_{i, k} / D_{i, k}-1\right\}}{\left\{S_{i-1, k} / D_{i-1, k}-1\right\}}
$$

it can be shown that

$$
\text { (2.5) } \frac{S_{i, k}}{I)_{i, k}}=\frac{\{n+1-q+2(i-k-1)\}\{n-1+q+2(k-i)\}\{n-q+2(i+k-1)\}\{2(k+i)-n-q\}}{\{n+1-q+2(i+k-1)\}\{n-q+2(i-k)\}\{n+q+2(k-i)\}\{2(k+i-1)-n+1-q\}}
$$

and

(26) $\frac{D)_{i, k}}{D_{i-1, k}}=\frac{\{n-q+2(i+k-2)\}\{n+q+2(k-i)\}\{n-1-q+2(i-k-1)\}\{2(k+i)-n-1-q\}}{\{n-q+2(i-k-1)\}\{2(k+i)-n-q\}\{n+1+q+2(k-i-1)\}\{n-1-q+2(i+k-1)\}}$.

The quantities in (23) can then be recursively calculated using the relation

$$
\frac{U_{i, k}}{U_{r-1-k, k}}=\frac{U_{i-1, k}}{U_{r-1-k, k}} \frac{U_{i, k}}{U_{i-1, k}}
$$

\begin{tabular}{|c|c|c|c|c|}
\hline \multirow[t]{2}{*}{ Formula } & \multicolumn{4}{|c|}{ Number of points used } \\
\hline & 7 & 15 & 31 & 63 \\
\hline $\begin{array}{l}\text { Gauss } \\
\text { Curtiss-Clenshaw } \\
\text { Tables M10-M13 }\end{array}$ & $\begin{array}{l}.118 \\
.254 \\
.132\end{array}$ & $\begin{array}{l}1.12 \times 10^{-3} \\
3.01 \times 10^{-3} \\
2.07 \times 10^{-3}\end{array}$ & $\begin{array}{l}6.75 \times 10^{-8} \\
9.95 \times 10^{-7} \\
3.99 \times 10^{-7}\end{array}$ & $\begin{array}{l}1.31 \times 10^{-21} \\
5.73 \times 10^{-12} \\
1.20 \times 10^{-14}\end{array}$ \\
\hline
\end{tabular}

with $i=r-k, \cdots, r-1$ in steps of one for each of $k=1,2, \cdots, r-2$. The calculations again show that the $g_{i}$ do not vary greatly in magnitude, and the roots of $W_{n-1}(x)$ can be calculated with little loss of accuracy due to cancellation.

TABLE 1

Davis-Rabinowitz $\sigma_{R}$ for $a=1.05$

2.3. The Extension of General Quadrature Formulae. The methods described in Sections 2.1 and 2.2 for the extension of the Gaussian and Lobatto formulae are 
specific in that they make use of a knowledge of the properties of the polynomial whose zeros are the abscissae of the quadrature formula. In general no useful properties may be known and it is necessary to resort to an alternative technique.

The basic equation (2) can be written in the equivalent form,

$$
\int_{-1}^{1} G_{n+p}(x) P_{k}(x) d x=0, \quad k=0,1, \cdots, p-1,
$$

where $P_{k}(x)$ is the Legendre polynomial. $G_{n+p}(x)$ can however be expanded as

$$
G_{n+p}(x)=\sum_{i=0}^{n+p} t_{i} P_{i}(x)
$$

and substitution in (28) gives

$$
\sum_{i=0}^{n+p} t_{i} \int_{-1}^{1} P_{i}(x) P_{k}(x) d x=0, \quad k=0,1, \cdots, p-1 .
$$

It is clear that this implies, due to the orthogonality properties of the Legendre polynomials, that $t_{i}=0$ for $i=0,1, \cdots, p-1$. Thus (29) becomes

$$
G_{n+p}(x)=\sum_{i=p}^{n+p} t_{i} P_{i}(x) .
$$

Taking account of the symmetry of the abscissae, (31) may be rewritten as

$$
G_{n+p}(x)=\sum_{i=1}^{[n / 2]+1} c_{i} P_{2 i-2+p+q}(x)
$$

where again $q=n-2[n / 2]$. Since the original abscissae $x_{j}, j=1, \cdots, n$ are roots of $G_{n+p}(x)$ and since $c_{[n / 2]+1}$ may be arbitrarily taken to be unity, then

$$
\sum_{i=1}^{[n / 2]} c_{i} P_{2 i-2+p+q}\left(x_{j}\right)=-P_{n+p}\left(x_{j}\right), \quad j=1,2, \cdots,\left[\frac{n}{2}\right] .
$$

The symmetry of the abscissae about the origin has also been taken into account in (33). The coefficients $c_{1}, c_{2}, \cdots, c_{[n / 2]}$ may be found by solving the $[n / 2]$ simultaneous equations which comprise (33) and hence the $p$ additional abscissae determined as the zeros of $G_{n+p}(x)$ as given by (32).

When this analysis is applied to the Gauss and Lobatto formulae, it can be shown that $p$ must be at least $n+1$ in the former case and $n-1$ in the latter case. The reason for this is that if $a_{1}, a_{2}, \cdots, a_{p}$ denote the abscissae added to the $n$-point Gauss formula, then the weight associated with $a_{j}$ in the resulting quadrature formula is (cf. [3])

$$
\omega_{j} \propto \int_{-1}^{1} P_{n}(x) S_{j}(x) d x
$$

where

$$
S_{j}(x)=\prod_{i=1 ; i \neq j}^{p}\left(x-a_{i}\right)
$$




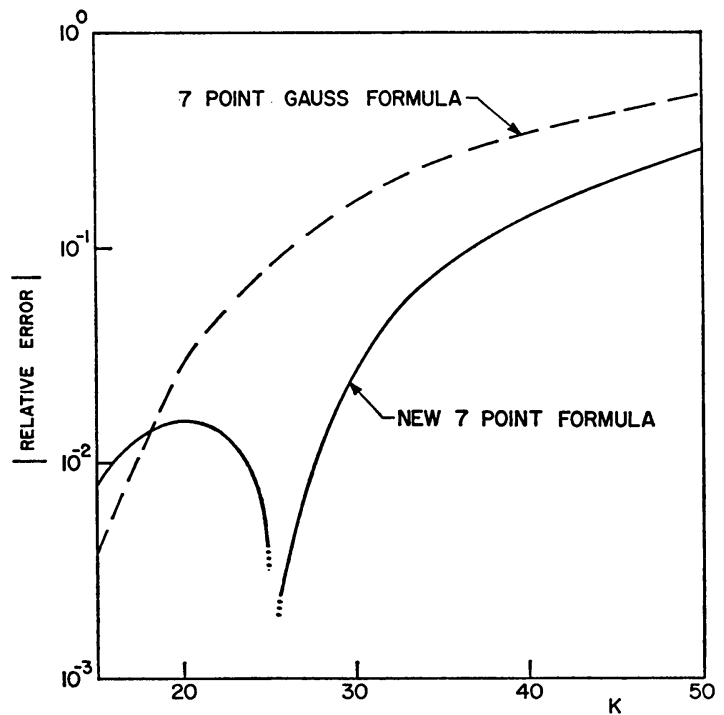

Figure 1. Absolute relative error in integrating $\int_{-1}^{1} x^{K} d x$ using the formula of Table M10. The corresponding result for the Gauss formula is also shown.

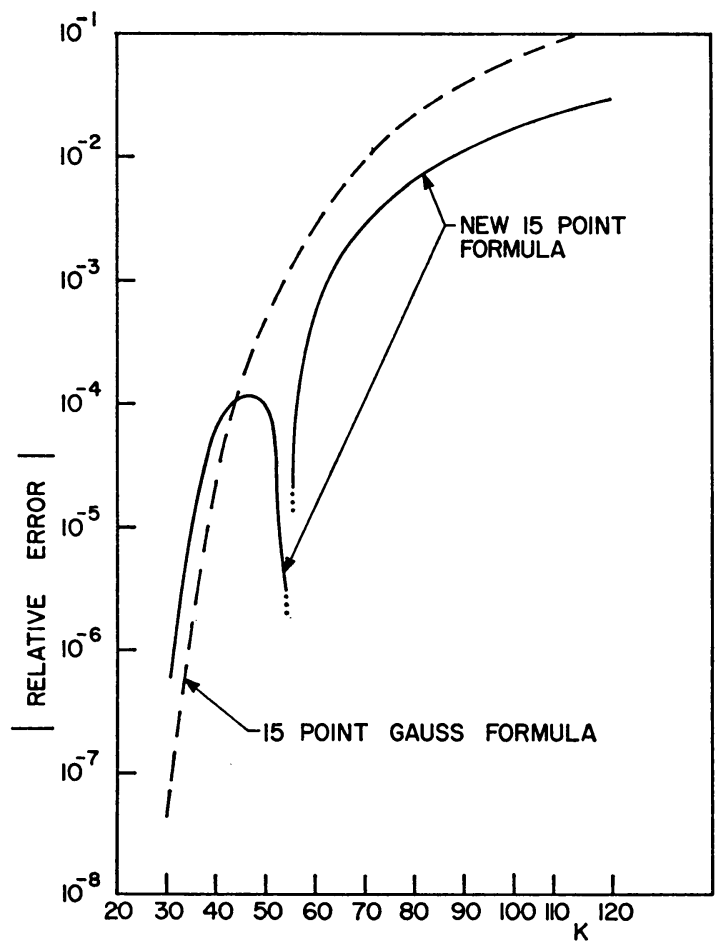

FigUre 2. Absolute relative error in integrating $\int_{-1}^{1} x^{K} d x$ using the formula of Table M11. The corresponding result for the Gauss formula is also shown. 
Noting that $S_{j}(x)$ is of degree $p-1$ and that $P_{n}(x)$ is orthogonal to all polynomials of degree less than $n$ then $p$ must be greater than $n$ otherwise $\omega_{j}$ would be zero. A similar argument extends to the $n$-point Lobatto formula whose abscissae are the roots of $\left[P_{n}(x)-P_{n-2}(x)\right]$. In this case $p$ must exceed $n-2$. The formulae given in Sections 2.1 and 2.2 thus represent the minimum extensions of the Gauss and Lobatto formulae. It may be noted that the extension of the integrating power of the $n$-point Gauss formula to degree $3 n+1$ by the addition of $n+1$ points as discussed by Kronrod [1] is not a property restricted to the Gauss formulae. Any $n$-point formula irrespective of its original integrating degree will have its degree increased to $3 n+1$ by the addition of $n+1$ points by the method discussed in this section. An example of this will be given later.

3. Some Extended Quadrature Formulae. In this section some examples of the applications of the techniques discussed earlier will be given. It has tacitly been assumed in Section 2 that the roots of the polynomial which defines the additional abscissae for any quadrature formula are all real. It has not in fact been possible to derive general conditions under which this is assured and the procedure has been to apply the techniques assuming that real roots exist but numerically checking for the occurrence of imaginary roots. All calculations have been carried out using not less than thirty decimal digits and any formulae quoted are correct to all digits given. The usual checks of integration of powers were successfully carried out.

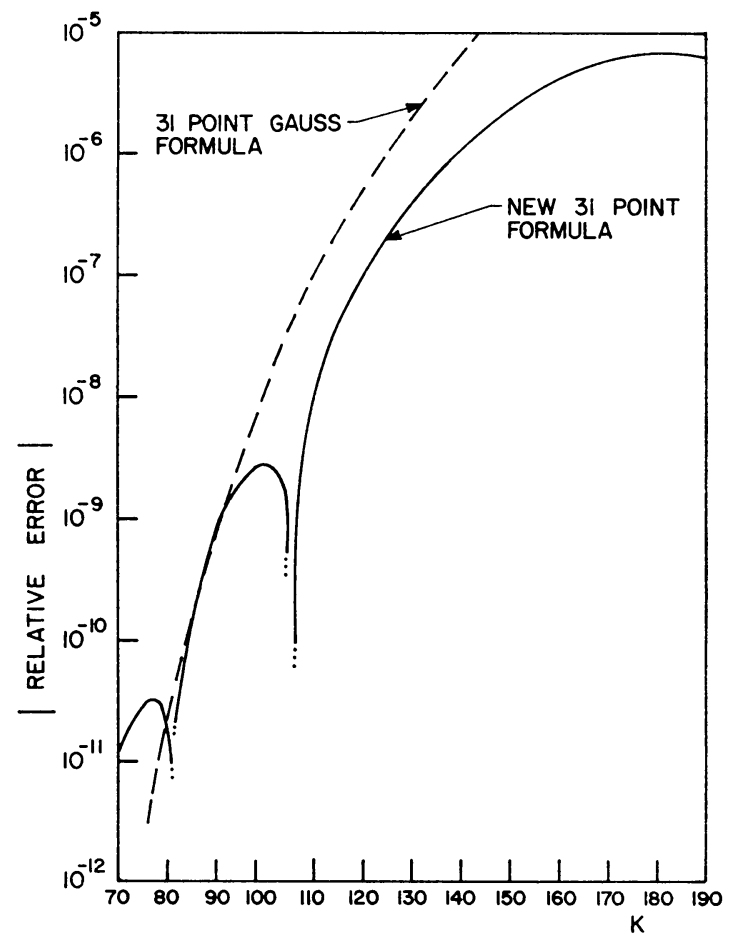

Figure 3. Absolute relative error in integrating $\int_{-1}^{1} x^{K} d x$ using the formula of Table M12. The corresponding result for the Gauss formula is also shown. 


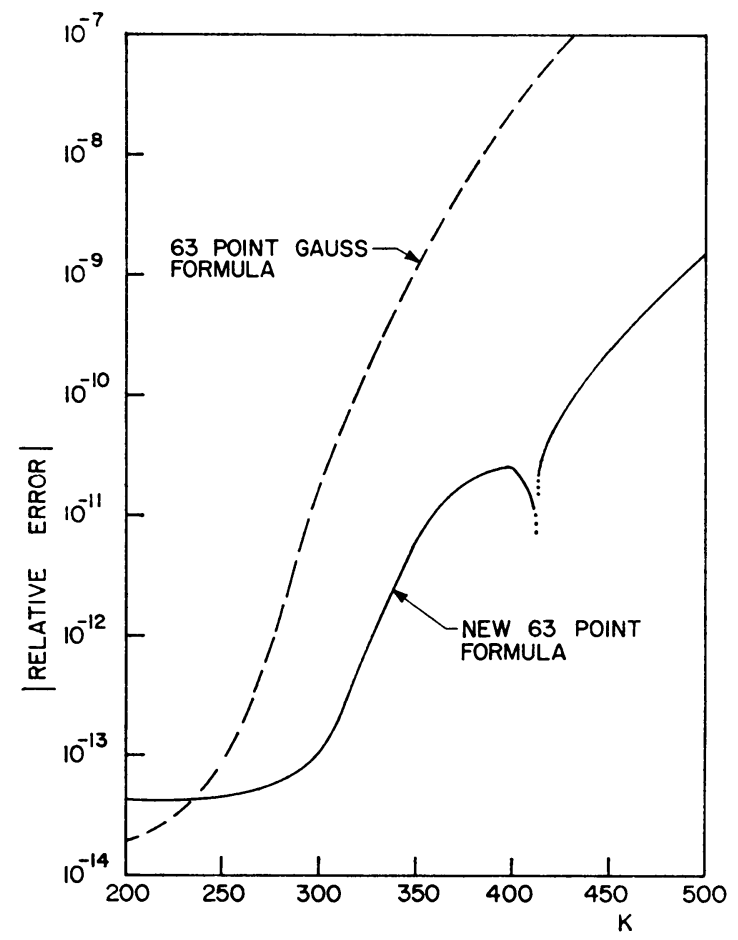

Figure 4. Absolute relative error in integrating $\int_{-1}^{1} x^{K} d x$ using the formula of Table M13. The corresponding result for the Gauss formula is also shown.

3.1. Gauss and Lobatto Formulae. In a recent paper [3] a set of economical quadrature formulae have been proposed which are based on the 65-point Gauss and 65-point Lobatto formulae. These formulae can be further extended by the addition of points as described in Sections 2.1 and 2.2. The weights and abscissae of the extended 65-point Gauss and 65-point Lobatto formulae, which are of respective degree 197 and 193 are given in Tables* M1 and M2. As further examples of the extension of Lobatto formulae, Tables M3 to M9 give the extended Lobatto formulae for $n=3$ to 9 .

3.2. General Formulae. Using the method of Section 2.3 a group of quadrature formulae were derived in the following sequence. Beginning with the 3-point Gauss formula, 4 abscissae were added to produce a 7 -point formula of degree 11. Then 8 abscissae were added to this formula to produce a 15-point formula of degree 23 . The process was continued until a 127-point formula of degree 191 was obtained. The effective degree of these $n$-point formulae is $(3 n-1) / 2$. The weights and abscissae for these formulae are given in Tables M10 to M14. It may be noted that the weights associated with all these formulae are positive so that they are likely to converge in a satisfactory manner.

A detailed assessment was made of the integrating power of the first four of the new formulae of degree $(3 n-1) / 2$. Figs. $1-4$ show the results obtained when they are applied to integrate powers of $x$ which they would not be expected to

* The letter M preceding a table number refers to the microfiche card. 
integrate exactly. The absolute relative error (defined as $\left|\left(I-I_{t}\right) / I_{t}\right|$ where $I_{\iota}$ is the true value of the integral and $I$ is the value obtained by the formula) is plotted against $K$, the power of $x$ being integrated. The results for the Gauss formulae using the same number of points are also shown for comparison. The sharp dips that appear on the curves are a result of a sign change in the relative error which give the formulae superior integrating performance to the Gauss formulae for high powers.

The performance of the new formulae has also been assessed by applying them to a large number of badly behaved integrands such as those having near singularities, cusps or singularities in their derivatives. In general it was found that the formulae had the important property of converging uniformly towards the true values of the integrals and were more accurate than the Gauss formula using the same number of points.

The quantities, $\sigma_{R}$, introduced by Davis and Rabinowitz [4] have also been calculated. An upper bound to the error $E$ of a quadrature formula can be expressed as

$$
|E| \leqq \sigma_{R}\|f\|
$$

where $\|f\|$ is the norm of the integrand over a region $R$ of the complex plane containing the range of integration. Table 1 shows the values of $\sigma_{R}$ obtained for the new formulae of Tables M10 to M13 together with the values of $\sigma_{R}$ for the Gauss and Curtiss-Clenshaw [5] formulae using the same number of points. The region $R$ has been taken as an ellipse with semimajor axis $a$ and semiminor axis $\left(a^{2}-1\right)^{1 / 2}$ with $a=1.05$. As a further comparison it may be noted that the Romberg formulae using $5,9,17$ and 33 points have $\sigma_{R}$ respectively equal to $1.24,0.422,0.102$, and 0.0155 ,

It should be emphasized again that the abscissae of these new formulae interlace with one another so that no computational labor is lost in going from a particular formula to the one of next higher degree. In practice, the successive application of the formulae would be used to monitor the convergence of the integral to which they are applied so that they are well suited to automatic quadrature. It thus appears that these new formula may form the basis of a very powerful technique for economically carrying out numerical integration.

Southwest Center for Advanced Studies

Dallas, Texas 75230

1. A. S. Kronrod, Nodes and Weights of Quadrature Formulas, English transl. from Russian, Consultants Bureau, New York, 1965. MR 32 \#597. 1931 .

2. E. W. Hobson, Spherical and Ellipsoidal Harmonics, Cambridge Univ. Press, New York,

3. T. N. L. PatTerson, "On some Gauss and Lobatto based quadrature formulae," Math. Comp., v. 22, 1968, pp. 877-881.

4. P. J. Davis \& P. R. Rabinowitz, "On the estimation of quadrature errors for analytical functions," Math. Comp., v. 8, 1954, pp. 193-'203. MR 16, 404.

5. C. W. Clenshiw \& A. R. Curtiss, "A method for numerical integration on an automatic computer," Numer. Math., v. 2, 1960, pp. 197-205. MR 22 \#8659. 